\title{
Reference Trajectory Tracking for Locally Designed Coherent Quantum Controls
}

\author{
Mazyar Mirrahimi,,,$+ \hbar$ Gabriel Turinici,,$\$$ and Pierre Rouchon ${ }^{\dagger}$ \\ Ecole des Mines de Paris, Centre Automatique et Systèmes, 60 Bd Saint-Michel, 75272 Paris cedex 06, France, \\ and INRIA Rocquencourt, Domaine de Voluceau, Rocquencourt B. P. 105, 78153 Le Chesnay Cedex, France, \\ and CERMICS-ENPC, Champs sur Marne, 77455 Marne la Vallée Cedex, France
}

Received: June 24, 2004; In Final Form: November 18, 2004

\begin{abstract}
Local time control methods are used in the simulation of quantum control phenomena because they conveniently ensure an increase of a predefined performance index and also avoid singularities associated with tracking procedures. However, the drawback of the existing implementations is that they only take into account onephoton, direct transitions and may stop at nonoptimal values of the index. We propose in this paper a modification of the currently used algorithms that addresses this issue and explain how the convergence is improved. Furthermore, when iterations are required, we show that this approach can be inserted into a monotonically convergent algorithm.
\end{abstract}

\section{Introduction}

Using lasers (or other external interactions) to influence the dynamical properties of quantum systems has been successfully demonstrated both theoretically, $51,23,24,28$ and in laboratory practice. ${ }^{1-4,11,13,35}$ Very often, control of quantum phenomena is expressed as the minimization of a setting-dependent cost functional that describes the goal to be attained and the eventual penalties to consider. Three types of generic minimization procedures have been used in the literature: stochastic iterative approaches (e.g., genetic algorithms), ${ }^{7,15}$ iterative critical point methods that use adjoint state information and give rise to monotonic algorithms, ${ }^{10,17,29,32,36}$ and tracking or local control procedures $6,8,12,16,20,30,31$ that obtain explicitly the control field from the prescribed trajectory that the system is required to take (and devise additional techniques to avoid eventual singularities). The advantage of this last class of methods is that it only requires one (or few) propagations of the time-dependent Schrödinger equation (TDSE); when larger systems are to be treated, this property may prove crucial for the numerical tractability of the simulations.

A recent example of the local control procedure ${ }^{30}$ defines a performance index in terms of the system's wave function or density matrix operator and of the target observables. The controlling field is then obtained through the requirement that this index increases monotonically during the optimization. Although successful application of this approach has been demonstrated for the control of the one-dimensional hydrogen fluoride (HF) molecule, no analysis is available to quantify the performance of this procedure in general circumstances. Starting from a study of the stopping points of the local control method, we were able to identify the ingredients that lead to successful convergence. For instance, it was observed that the scheme only

* To whom correspondence should be addressed: Telephone: +33-1-40-51-91-64. Fax: +33-1-40-51-91-65. E-mail: mazyar.mirrahimi@ ensmp.fr.

† Ecole des Mines de Paris, Centre Automatique et Systèmes, 60 Bd Saint-Michel, 75272 Paris cedex 06, France.

$\doteqdot$ CERMICS-ENPC, Champs sur Marne, 77455 Marne la Vallée Cedex, France.

$\S$ INRIA Rocquencourt, Domaine de Voluceau, Rocquencourt B. P. 105, 78153 Le Chesnay Cedex, France. exploits direct, one-photon-coupling capabilities of the dipolemoment operator. When full direct coupling between all eigenstates of the free Hamiltonian is not available, it will be "trapped" into local minima on its way to convergence. However, supposing existence of complete first-order transitions is a restrictive assumption, and some standard benchmark cases $^{27,33}$ do not fall within this class.

To improve this behavior, we document in this paper a new procedure that exploits transitions at all orders; this approach also successfully treats systems with degenerate transitions, not always covered by the initial approach. In addition, an enhancement of this scheme that treats situations with constraints on the total time or coupling field intensity is proposed. This algorithm is shown to display monotonic convergence behavior.

The paper is organized as follows: we introduce in section 2 our Lyapunov-based method and compare its convergence properties with those of the previously proposed schemes. We illustrate these results with numerical simulations in section 3 . Further extensions of the method are given in subsection 4 . Discussions and concluding remarks are presented in section 5 .

\section{Lyapunov-Based Designs}

Consider the control via an external interaction (e.g., a laser field) of the expectation value of a physical observable operator $O$ in a quantum system. The system's wave function is governed by the Schrödinger equation

$$
i \frac{\partial}{\partial t} \Psi=\left.\left[H_{0}-\mu \epsilon(t)\right] \Psi \quad \Psi\right|_{t=0}=\Psi_{0}
$$

where $\Psi_{0}$ is the initial state, $H_{0}$ is the internal Hamiltonian, $\mu$ is the coupling dipole moment, and $\epsilon(t)$ is the external field intensity. The control objective is to maximize the expectation value of the operator $O$. We will keep the wave-function description throughout the paper, but similar considerations apply to the density matrix formalism. When relevant, we will indicate the analogous results than can be obtained in that setting.

This problem has been treated in a general form in ref 6 , where the control objective is to guide the dynamical trajectory 
such that the expectation value of $O$ follows a desirable track, $S(t):\langle\Psi(t)|O| \Psi(t)\rangle=S(t)$. The approach of ref 6 , named as tracking, has also been studied in refs 8 and 16. It consists of obtaining the control field from the evolution equation of $S(t)$ and the requirement that $S(t)$ follows exactly a specified trajectory. As a typical problem for such reverse-design techniques, singularities may arise in the inversion process. These singularities are classified and studied in refs 25 and 26. A method to treat these singularities is presented in ref 26.

Another method of the same nature has been introduced in ref 31, where locally optimized control fields are designed for the control of quantum dynamics. The optimization theory of LTI (linear time-invariant) systems has been used in small intervals of time to design the control field in a quite direct manner.

Finally, in refs 12 and 30, a first approach based on Lyapunov techniques has been introduced. The method consists of using a performance index $y(t)$ that formulate the desired physical properties to be satisfied by the system, defined as

$$
y(t)=y\left(\left\langle\tilde{O}_{1}(t)\right\rangle,\left\langle\tilde{O}_{2}(t)\right\rangle, \ldots,\left\langle\tilde{O}_{N}(t)\right\rangle\right)
$$

Here, $\left\langle\tilde{O}_{j}(t)\right\rangle$ for $j \in\{1,2, . ., N\}$ denotes the expectation value of the physical observables given by the Hermitian operators $\tilde{O}_{j}(t)$ that evolve with the equation of motion

$$
\frac{\mathrm{d}}{\mathrm{d} t} \tilde{O}_{j}(t)=i\left[\tilde{O}_{j}, H_{0}\right]
$$

The controlling field is then chosen so that it ensures the increase of the performance index $y(t)$. A simple computation shows that

$$
\frac{\mathrm{d} y(t)}{\mathrm{d} t}=-\epsilon(t) \sum_{i=1}^{N} \frac{\partial y(t)}{\partial\left\langle\tilde{O}_{j}(t)\right\rangle}\left\langle\left[\tilde{O}_{j}(t), \mu / i\right]\right\rangle
$$

Thus, any feedback of the form

$$
\epsilon(t)=K\left(t,-\sum_{i=1}^{N} \frac{\partial y(t)}{\partial\left\langle\tilde{O}_{j}(t)\right\rangle}\left\langle\left[\tilde{O}_{j}(t), \mu / i\right]\right\rangle\right)
$$

where $K(t, s)=\mathbb{R} \times \mathbb{R} \rightarrow \mathbb{R}$ is a smooth function such that

$$
s K(t, s) \geq 0 \forall s \in \mathbb{R} \quad K(t, s)=0 \Leftrightarrow s=0
$$

ensures $\mathrm{d} y(t) / \mathrm{d} t \geq 0$. In the particular case when $y(t)$ is an expectation value of the projection operator $|\phi\rangle\langle\phi|$ to an eigenstate $\phi$ of the internal Hamiltonian, this is equivalent to choose a feedback design of the form

$$
\epsilon(t)=K(t, \operatorname{Im}(\langle\mu \Psi \mid \phi\rangle\langle\phi \mid \Psi\rangle))
$$

As we will see in the formal analysis of this section and in the numerical simulations of the next section, this Lyapunov-based control only takes in account the first-order transitions. In the case where all of the eigenstates of $H_{0}$ are coupled via the dipole moment $\mu$, this control field appears to be efficient. However, in the case where some states of the system are not directly coupled but transitions of higher orders exist, the design will fail to lead the system toward the target state. It is shown in ref 18 that the trajectories of the system will converge toward a subspace, with a dimension strictly more than one, of the system's space. Numerical simulations justifying this claim are presented in the next section.

To cure these drawbacks, we introduce here another version of this method that enables the use of multiphotonic transitions. We present this approach for the general formulation of the local coherent control theory and give some numerical simulations for the particular case of the projection operators. Our procedure automatically identifies the multiphoton transition paths needed to reach the target. Once the driving field is obtained, an a posteriori analysis of the system dynamics (obtained during the resolution of the TDSE) can inform on the control mechanisms that were found useful.

2.1. Control Design. Even if the operators $\tilde{O}_{j}$ are not constants of time, they are invariants of the free system without any control term. ${ }^{14}$ The idea consists in defining reference operators whose time dependence with respect to the free system is not stationary and which becomes equal to desired $O_{j}$ after a large time $T$. The formal analysis of the next subsection shows how using such nonstationary reference operators allows to use higher order transitions.

Take observable operators $\tilde{O}_{j}(t)$ whose dependence with respect to time is given by the following equation of motion:

$$
\begin{gathered}
\frac{\mathrm{d} \tilde{O}_{j}}{\mathrm{~d} t}=i\left[\tilde{O}_{j}, H_{0}-f(t) \mu\right] \\
\tilde{O}_{j}(t=T)=O_{j}
\end{gathered}
$$

where $f(t)$ is a reference field not identically zero and such that $f(t)=0$ for all $t \geq T$. Deriving formally eq 2, we obtain

$$
\begin{gathered}
\frac{\mathrm{d} y(t)}{\mathrm{d} t}=\sum_{i=1}^{N} \frac{\partial y(t)}{\partial\left\langle\tilde{O}_{j}(t)\right\rangle} \frac{\mathrm{d}\left\langle\tilde{O}_{j}(t)\right\rangle}{\mathrm{d} t} \\
=-(\epsilon(t)-f(t)) \sum_{i=1}^{N} \frac{\partial y(t)}{\partial\left\langle\tilde{O}_{j}(t)\right\rangle}\left\langle\left[\tilde{O}_{j}(t), \mu / i\right]\right\rangle
\end{gathered}
$$

Thus, any feedback of the form

$$
\epsilon(t)=f(t)+K\left(t,-\sum_{i=1}^{N} \frac{\partial y(t)}{\partial\left\langle\tilde{O}_{j}(t)\right\rangle}\left\langle\left[\tilde{O}_{j}(t), u / i\right]\right\rangle\right)
$$

where $K(t, s)$ verifies the same conditions as before, will increase the value of the performance index $y(t)$.

Now let us consider the case where the performance index is the expectation value of a physical observable operator. We take

$$
y(t)=\langle\tilde{O}(t)\rangle
$$

where $\tilde{O}(t)$ verifies eq 5 with a final condition $\tilde{O}(t=T)=O_{f}$. One important question to ask is when does the system stop before reaching the maximum value of the performance index.

The reference operator $\tilde{O}(t)$ reaches the desired observable operator $O_{f}$ at time $T$ (supposed to be large enough), and thus, the control of $\left\langle O_{f}\right\rangle$ terminates successfully unless $\langle\tilde{O}(t)\rangle$ stops increasing toward its maximal value. For a rigorous proof of a convergence result for the case of a projection operator, under some technical but not restrictive assumptions on $f(t)$, see ref 19. However, to illustrate the arguments of the proof, let us explore formally the simple case where the reference trajectory corresponds to the driving field $f(t) \equiv 0$, which is the method 
proposed in ref 30 . We will make clear when using this particular field $[f(t) \equiv 0]$ can the expectation value of the physical observable $\tilde{O}$ reach its maximal value and when this field fails to give a convenient reference observable operator. Then, we will explain how using a nonstationary well-chosen reference field one can overcome this lack of convergence (the analysis for the case of a general index depending on the mean value of many physical observables could be treated along the same lines).

2.2. Convergence Analysis. Suppose that the reference driving field is zero, $f \equiv 0$. Formally the mean value $\langle\tilde{O}\rangle$ stops increasing when the control field $\epsilon(t)$ becomes uniformly equal to the reference driving field $f(t)$, which happens if and only if $\tilde{\epsilon}(t)=\epsilon(t)-f(t)$ and all of its derivatives are zero. This may be written as

$$
\left\langle\left[\tilde{O}, a d_{\mu / i}^{k} \frac{H_{0}}{i}\right]\right\rangle=\left\langle\Psi\left|\left[\tilde{O}, a d_{\mu / i}^{k} \frac{H_{0}}{i}\right]\right| \Psi\right\rangle=\underset{ }{0} \begin{aligned}
& \forall k=0,1,2, \ldots
\end{aligned}
$$

Here, $a d_{Z}^{\mathrm{m}}(Y)$ is a notation for iterative commutators

$$
a d_{Z}^{0}(Y)=Z \quad a d_{Z}^{\mathrm{m}}(Y)=\left[Y, a d_{Z}^{m-1}(Y)\right] \text { for } m \geq 1
$$

In fact $\tilde{\epsilon}=0$ implies $\langle[\tilde{O}, \mu / i]\rangle=0$. In general, we can prove that

$$
\frac{\mathrm{d}^{j}}{\mathrm{~d} t_{j}} \tilde{\epsilon}=(-1)^{j}\left\langle\left[\tilde{O}, a d_{\mu l i}^{j} \frac{H_{0}}{i}\right]\right\rangle \text { for } j=0,1,2, . .
$$

Indeed, suppose that eq 7 holds for $j \leq k$, then

$$
\begin{gathered}
\left.\frac{\mathrm{d}^{k+1}}{\mathrm{~d} t^{k+1}} \tilde{\epsilon}=(-1)^{k} \frac{\mathrm{d}}{\mathrm{d} t} /\left[\tilde{O}, a d_{\mu / i}^{k} \frac{H_{0}}{i}\right]\right\rangle \\
\left.=(-1)^{k} /\left[\left[\tilde{O}, a d_{\mu / i}^{k} \frac{H_{0}}{i}\right], \frac{H_{0}}{i}\right]\right\rangle+(-1)^{k}\left\langle\left[\frac{\mathrm{d}}{\mathrm{d} t} \tilde{O}, a d_{\mu / i}^{k} \frac{H_{0}}{i}\right]\right\rangle \\
\left.\left.=(-1)^{k} /\left[\left[\tilde{O}, a d_{\mu / i}^{k} \frac{H_{0}}{i}\right], \frac{H_{0}}{i}\right]\right\rangle-(-1)^{k} /\left[\left[\tilde{O}, \frac{H_{0}}{i}\right], a d_{\mu / i}^{k} \frac{H_{0}}{i}\right]\right\rangle \\
\left.\left.\left.\left.=(-1)^{k} /\left[\left[\tilde{O}, a d_{\mu / i}^{k} \frac{H_{0}}{i}\right], \frac{H_{0}}{i}\right]\right\rangle+(-1)^{k}\right\rangle\left[\frac{H_{0}}{i}, \tilde{O}\right], a d_{\mu / i}^{k} \frac{H_{0}}{i}\right]\right\rangle \\
=(-1)^{k+1}\left\langle\left[\left[a d_{\mu / i}^{k} \frac{H_{0}}{i}, \frac{H_{0}}{i}\right], \tilde{O}\right]\right\rangle \\
\left.=(-1)^{k+1} /\left[a d_{\mu / i}^{k+1} \frac{H_{0}}{i}, \tilde{O}\right]\right\rangle
\end{gathered}
$$

where we have used the Jacobi identity for the Lie brackets.

We write the system in the eigenbasis corresponding to the hermitian matrix $H_{0}$. Thus, $H_{0}$ is diagonal, $H_{0}=$ diag$\left(\lambda_{1}, \ldots, \lambda_{n}\right)$. Then, the commutator $\left[H_{0}, B\right]$ for any $n \times n$ matrix $B=\left(B_{i j}\right)$ is $\left[H_{0}, B\right]=\left[\left(\lambda_{i}-\lambda_{j}\right) B_{i j}\right]_{i, j}$, and thus, for any $k \geq 1$

$$
a d_{\mu / i}^{k} \frac{H_{0}}{i}=\left(\frac{1}{i}\right)^{k+1}\left[\left(\lambda_{i}-\lambda_{j}\right)^{k} \mu_{i j}\right]
$$

Using eq 7, eq 6 becomes equivalent to

$$
\begin{gathered}
\sum\left(\lambda_{i}-\lambda_{j}\right)^{k} \mu_{i j}\left(\bar{\Psi}_{i} \Phi_{j}+\bar{\Psi}_{j} \Phi_{i}-\bar{\Phi}_{j} \Psi_{i}-\bar{\Phi}_{i} \Psi_{j}\right)=0 \\
\forall k \in\{0,2,4, . .\} \\
\sum\left(\lambda_{i}-\lambda_{j}\right)^{k} \mu_{i j}\left(\bar{\Psi}_{i} \Phi_{j}-\bar{\Psi}_{j} \Phi_{i}+\bar{\Phi}_{j} \Psi_{i}-\bar{\Phi}_{i} \Psi_{j}\right)=0 \\
\forall k \in\{1,3,5, . .\}(9)
\end{gathered}
$$

where $\Phi=\bar{O} \Psi$. Then, under the assumption ${ }^{34}$

A1: $H_{0}$ does not have degenerate transitions, i.e., $\lambda_{i}-\lambda_{j} \neq$ $\lambda_{a}-\lambda_{b}$, for $(i, j) \neq(a, b)$.

equation 9 becomes equivalent to

$$
\mu_{i j}\left(\bar{\Psi}_{j} \Phi_{i}-\Psi_{i} \bar{\Phi}_{j}\right)=0 \quad \forall i \neq j \in\{1,2, . ., n\}
$$

So if

A2: for any $i \neq j \in\{1,2, . ., n\}$, the coefficient $\mu_{i j}$ does not vanish $\left(\mu_{i j} \neq 0\right)$.

then, $\Phi$ must be proportional to $\Psi$, which means that $\Psi$ must be an eigenstate of the physical observable operator $\tilde{O}$. Therefore, under the assumptions A1 and A2, the algorithm stops converging toward the maximal value of the expectation value of $\tilde{O}$ only on eigenstates of the operator $\tilde{O}$. However, because only the eigenstate of $\tilde{O}$ with a maximum eigenvalue is stable, the system stops only on this eigenstate. For the case of the density matrix description, the same analysis leads us to conclude that stopping points necessarily commute with the observable $\tilde{O}$.

Let us now consider the case where the assumption A2 is not fulfilled. It means that some states of the free Hamiltonian $H_{0}$ are not coupled by one-photon transitions, i.e., some $\mu_{i j}$ values are zero. This situation may arise for many controllable molecules. We will see that in such cases the algorithm with a reference field $f \equiv 0$ will not lead the system to the maximum value of $\langle\tilde{O}\rangle$.

To clarify the situation, let us restrict ourselves to the representative case where the operator $\tilde{O}$ is the projection operator on eigenstate $\phi$ of the internal Hamiltonian with energy $\lambda, \tilde{O}=|\phi\rangle\langle\phi|$.

A stable equilibrium manifold for the system is given by the vector space

$$
E=\operatorname{span}\left\{\phi_{i} ;\left\langle\phi|\mu| \phi_{i}\right\rangle=0 \quad H_{0} \phi_{i}=\lambda_{i} \phi_{i}\right\}
$$

Indeed, the feedback design for this observable is given by eq 4. Clearly, $E$ represents an equilibrium manifold for this field design; when we start from any state in $E$, the feedback design vanishes and the system's dynamic will be stationary. The mathematical analysis elaborated in ref 18 based on LaSalle's invariance principle shows that any trajectory of the closedloop system will converge toward an equilibrium point in this vector space and not necessarily toward $\phi$. Numerical simulations of the next section illustrate this fact for some usual and representative examples of finite dimension.

On the contrary, adding a nonzero reference driving field $f(t)$ will perturb the free Hamiltonian of the system and will lead the system to verify the assumptions $\mathbf{A 1}$ and A2. To have effective perturbations on the free Hamiltonian if we are in the weak fields regime, one should use resonant driving fields $f(t)$. In the case of strong fields this is not necessary. 

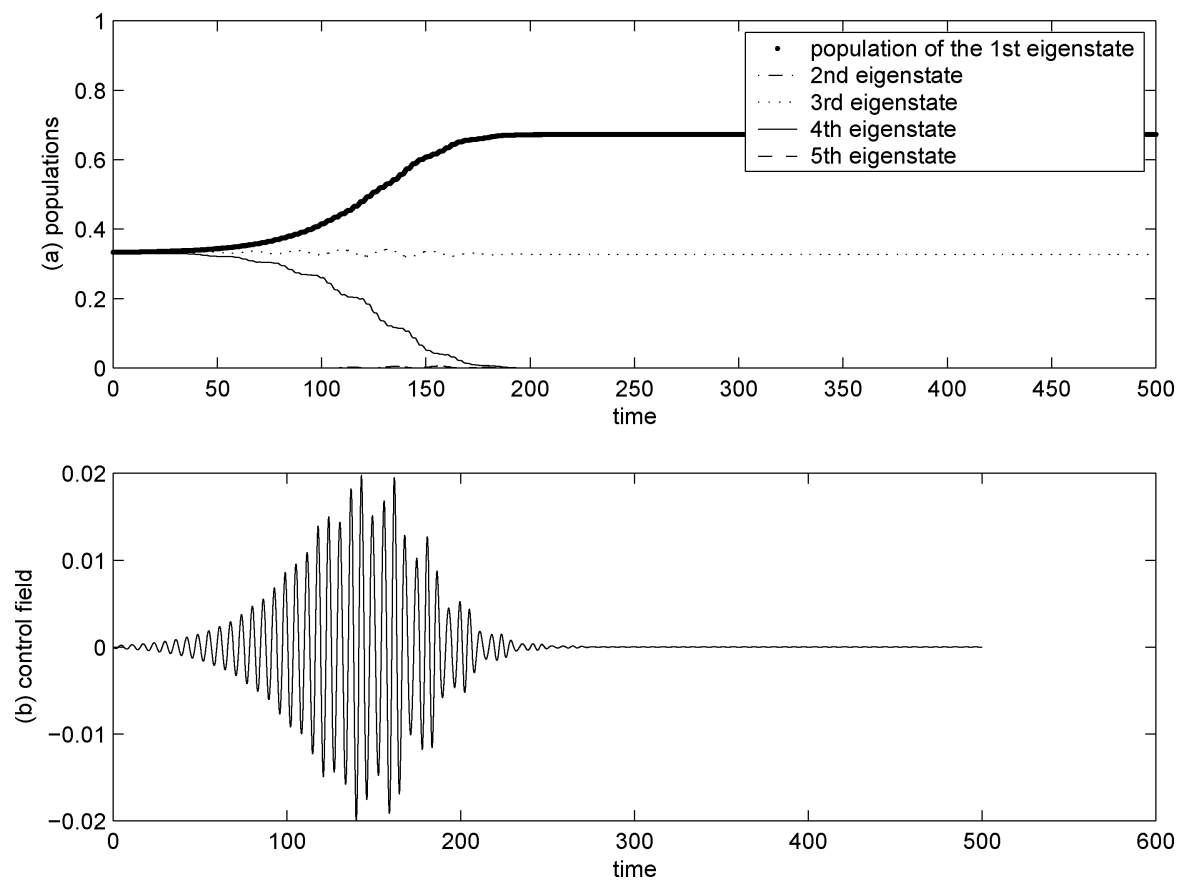

Figure 1. (a) Square of the norm of the projection of the system's trajectory $\Psi(t)$ with a feedback of the form in eq 4 on different eigenstates of the system, i.e., $\left|\left\langle\Psi(t) \mid \phi_{i}\right\rangle\right|^{2}$. (b) Control field found by eq 4 where $K(t, s)=\sigma(t) s$ with $\sigma(t)=1 / 4 \exp \left(-0.1 e-3(t-T / 2)^{2}\right)\left(5\right.$-level system). ${ }^{13}$

For example, when $O$ is a projection operator $O=|\phi\rangle\langle\phi|$ using a reference trajectory

$$
\begin{gathered}
i \frac{\partial}{\partial t} \Psi_{\mathrm{r}}=\left(H_{0}-f(t) \mu\right) \Psi_{\mathrm{r}} \\
\Psi_{\mathrm{r}}(T)=\phi
\end{gathered}
$$

and the Lyapunov-based method to obtain an increasing function $\left|\left\langle\Psi(t) \mid \Psi_{\mathrm{r}}(t)\right\rangle\right|^{2}$, the following simple design for the control field can be proposed:

$$
\epsilon(t)=f(t)+K\left(t, \operatorname{Im}\left(\left\langle\mu \Psi \mid \Psi_{\mathrm{r}}\right\rangle\left\langle\Psi_{\mathrm{r}} \mid \Psi\right\rangle\right)\right)
$$

Numerical simulations of the next section, which are done in this particular case, make clear how using a nonzero reference field causes the assumptions $\mathbf{A} 1$ and $\mathbf{A 2}$ to hold true.

\section{Numerical Simulations}

Consider the 5-level system (already used in the literature $)^{27,33,34}$ where the internal Hamiltonian $H_{0}$ and the dipole moment $\mu$ are

$$
H_{0}=\left(\begin{array}{lllll}
1.0 & 0 & 0 & 0 & 0 \\
0 & 1.2 & 0 & 0 & 0 \\
0 & 0 & 1.3 & 0 & 0 \\
0 & 0 & 0 & 2.0 & 0 \\
0 & 0 & 0 & 0 & 2.15
\end{array}\right) \mu=\left(\begin{array}{lllll}
0 & 0 & 0 & 1 & 1 \\
0 & 0 & 0 & 1 & 1 \\
0 & 0 & 0 & 1 & 1 \\
1 & 1 & 1 & 0 & 0 \\
1 & 1 & 1 & 0 & 0
\end{array}\right)
$$

This system is controllable: the Lie algebra spanned by $H_{0} / i$ and $\mu / i$ is $u(5) .{ }^{27}$ Let us suppose that the goal is to steer the system's wave function to the first eigenstate $\phi=(1,0,0,0$, $0)^{t}$ of $H_{0}$ with energy $\lambda=1$. Using the stationary projection operator corresponding to the first eigenstate $\tilde{O}(t)=|\phi\rangle\langle\phi|$, we obtain the following design for the control field:

$$
\epsilon(t)=K(t, \operatorname{Im}(\langle\mu \Psi \mid \phi\rangle\langle\phi \mid \Psi\rangle))={K\left(t, \operatorname{Im}\left(\Psi_{1}\left(\Psi_{4}^{*}(t)+\Psi_{5}^{*}(t)\right)\right)\right)}
$$

Suppose that the initial state is a linear combination of the first three eigenstates corresponding to the energies $1,1.2$, and 1.3. Evidently, for such initial state, the control field found by the Lyapunov design vanishes for all $t \geq 0$, and therefore, the system state will not converge toward the desired target state. Even if the population of the eigenstates $\phi_{4}$ and $\phi_{5}$ (which have direct transitions with $\phi_{1}$ ) in the initial state are not zero, the system's trajectory might converge to a state of the system in the eigenspace generated by $\phi_{1}, \phi_{2}$, and $\phi_{3}$, which turns out to be the stable equilibrium manifold of the system. The simulation in Figure 1 shows this fact, when the initial state is $1 / \sqrt{ } 3(1,0,1,1,0)$ and the function $K(t, s)$ is defined as

$$
K(t, s)=\sigma(t) s
$$

with $\sigma(t)$ being a Gaussian envelope centered at $T / 2=250$. Here and throughout the paper, we express the system's state in the eigenbasis of the internal Hamiltonian $H_{0}$. To solve the TDSE between two consecutive times $t$ and $t+\mathrm{d} t$, we compute the propagator $\exp \left(i\left(H_{0}-\epsilon(t) \mu\right) \mathrm{d} t\right)$. We refer to end of section 3 for a discussion on the numerical schemes to be used when infinite dimensional settings are treated.

As one can see the system's wave function $\Psi$ stops its progression to the target state $\phi_{1}$ when attaining only $60 \%$ of the population. Note that the algorithm with zero reference field $f(t) \equiv 0$ forces the control field $\epsilon(t)$ to vanish after the time $t=300$ because of the feedback term $\operatorname{Im}(\langle\mu \Psi \mid \phi\rangle\langle\phi \mid \Psi\rangle)$ that tends to zero [irrespective of the choice of $K(t, s)=$ $\sigma(t) s$; in fact, at $t=300, \sigma(t)$ takes values near its maximum reached at $t=T / 2]$. Simulations with any other choice of $K(t, s)$ lead to the same behavior. Moreover, if we increase the total simulation time $T$, the molecule will be "trapped" in the same state. 

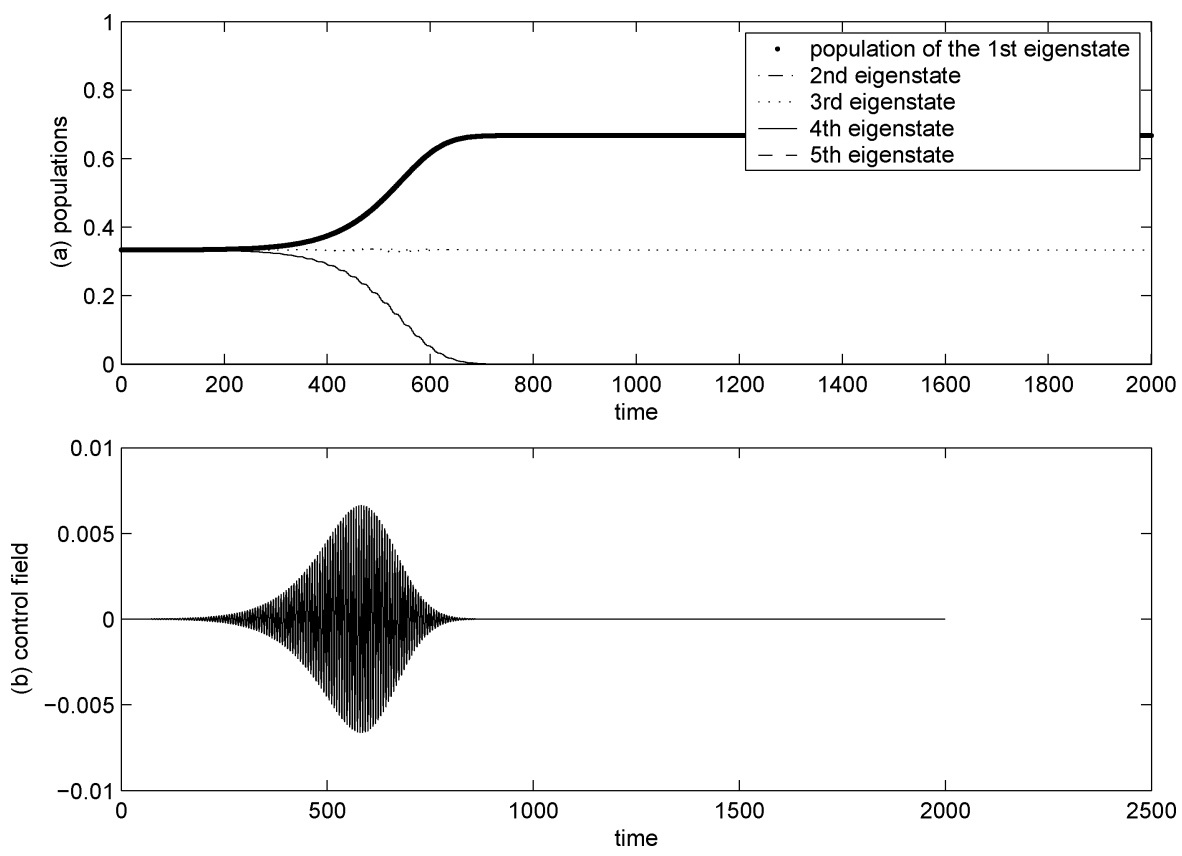

Figure 2. (a) Square of the norm of the projection of the system's trajectory $\Psi(t)$ using the performance index in eq 14 , on different eigenstates of the system. (b) Control field (5-level system). ${ }^{13}$


Figure 3. (a) Square of the norm of the projection of the reference trajectory $\Psi_{\mathrm{r}}(t)$ solution of 11. (b) Square of the norm of the projection of the system's trajectory $\Psi(t)$ using the feedback design of eq 12, on different eigenstates of the system. (c) Control field found by eq 12 where $f(t)=\sigma(t) \sin (t)$ and $K(t, s)=\sigma(t) s$ with $\sigma(t)=0.01 \exp \left(-0.1 e-4(t-T / 2)^{2}\right)(5$-level system $) .{ }^{13}$

In ref 30 , a method has been introduced to accelerate the convergence to the target state when this convergence is slow. It consists of using a performance index

$$
y(t)=\sum_{i=1}^{5} \alpha_{i}\left\langle P_{i}\right\rangle
$$

where $P_{i}=\left|\phi_{i}\right\rangle\left\langle\phi_{i}\right|$ is the projection operator on the $i$ th eigenstate of the free Hamiltonian and $\alpha_{1}$ is chosen to be bigger than other coefficients. This cure appears to be successful when the interactions between states are weak. However, in general, it has the same drawback as the last method. Let us see this fact in another simulation. We take the same initial condition $\Psi_{0}=1 / \sqrt{ } 3(1,0,1,1,0)$, and using the proportions $\left(\alpha_{1}, \alpha_{2}, \alpha_{3}\right.$, $\left.\alpha_{4}, \alpha_{5}\right)=(10,1,1,1,7)$, one obtains the result in Figure 2, which turns out to be quite similar to the last simulation. The feedback design $K(t, s)$ is chosen as before. Now let us consider a nonzero reference perturbing field $f(t)$. The simulation in Figure 3 corresponds to $f(t)=\sigma(t) \sin (t)$, where $\sigma(t)$ is a Gaussian envelope centered at $t=T / 2=1000$. The initial state is still $1 / \sqrt{ } 3(1,0,1,1,0)$, and the feedback design is $K(t, s)=\sigma(t) s$. Once again, the control field vanishes after the time $t=900$, and this is not because of the special form of $K(t, s)$ but because the feedback design converges to zero at the state where the molecule is "trapped".

As another situation where problems appear for a stationary reference field, one may consider a free Hamiltonian $H_{0}$ with a degenerate spectrum. We will consider here a 4-level 

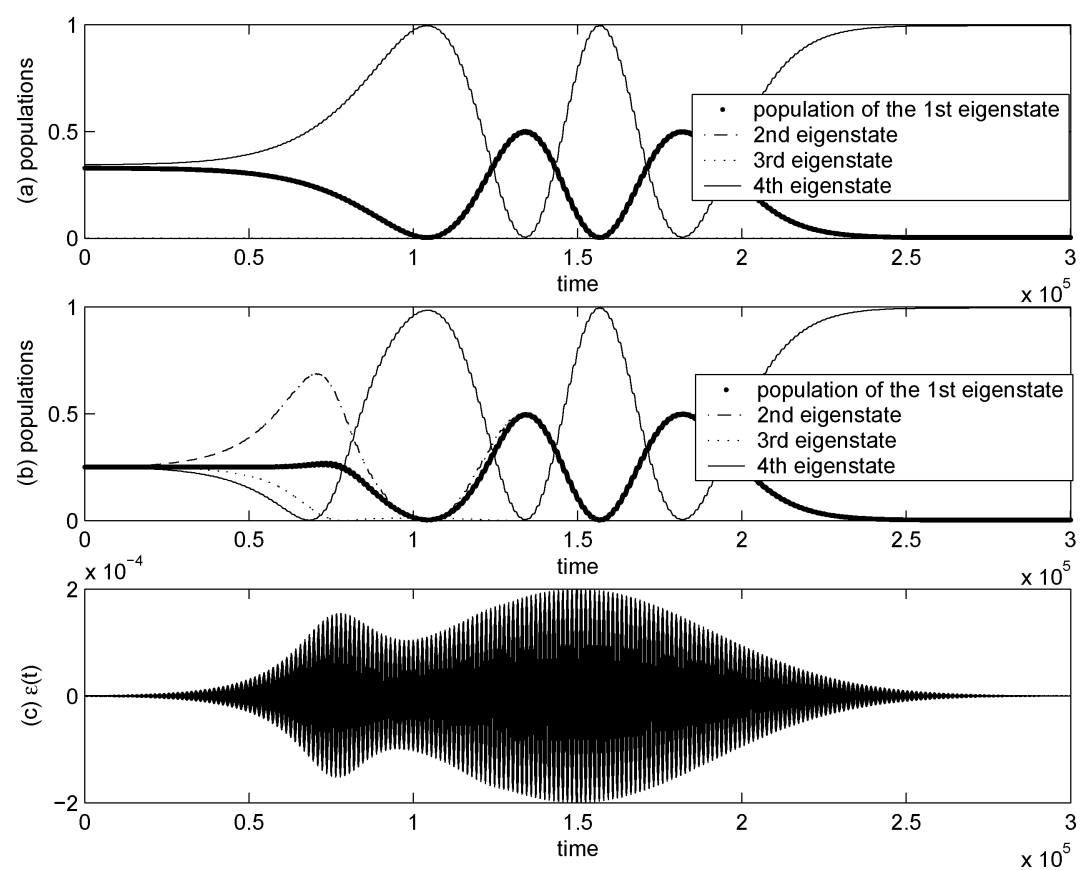

Figure 4. (a) Square of the norm of the projection of the reference trajectory $\Psi_{\mathrm{r}}(t)$ solution of 11. (b) Square of the norm of the projection of the system's trajectory $\Psi(t)$ using the feedback design of eq 12, on different eigenstates of the system. (c) Control field found by eq 12 where $f(t)=\sigma(t)\left(\sin \left(\omega_{1} t\right)+\sin \left(\omega_{2} t\right)\right.$ and $K(t, s)=10 \sigma(t) s$ with $\omega_{1}=0.095863, \omega_{2}=0.095683-0.004556$, and $\sigma(t)=0.1 e-3 \exp (-0.3 e-9(t-$ $\left.T / 2)^{2}\right)\left(4-\right.$ level system). ${ }^{15}$

system $^{9,}$ 21,22,34 (also see ref 28-page 152)

$\begin{aligned} \mathrm{H} 0= & \left.\begin{array}{lllll}0 & 0 & 0 & 0 \\ 0 & .004556 & 0 & 0 \\ 0 & 0 & 0.095683 & 0 & \\ 0 & 0 & 0 & 0.095 & 683\end{array}\right) \mu= \\ & \left(\begin{array}{lllll}0 & 1 & 1 & -1 \\ 1 & 0 & 1 & 1 \\ 1 & 1 & 0 & 0 \\ -1 & 1 & 0 & 0\end{array}\right)\end{aligned}$

The target state is $(0,0,0,1)$ in the eigenspace corresponding to the multiple eigenvalue 0.095683 . Figure 4 illustrates a simulation of this system when the open-loop part is given by a function $f=\sigma(t)\left(\sin \left(\omega_{1} t\right)+\sin \left(\omega_{2} t\right)\right.$, where $\sigma$ is a Gaussian envelope centered around $T / 2=1.5 e+5$ and $\omega_{1}$ and $\omega_{2}$ are transition frequencies. The initial state is set to be $\Psi_{0}=1 / 2(1$, $1,1,1)$, and the feedback design is $K(t, s)=10 \sigma(t) s$.

As noticed previously, both systems considered here are typical cases of finite dimensional systems already presented in the literature. The time and field intensity parameters found by the Lyapunov-based approach are coherent with values found by other algorithms.

Note that the exactly same design can be used for situations where the spectrum is continuous, and therefore, the system is infinitely dimensional. ${ }^{37}$ In this case, the numerical simulations are to be performed using, e.g., a third-order potential-centered split-operator method. ${ }^{38}$

\section{Further Extensions}

The procedure presented in section 2 extends the work of ref 30 that considers the case $f \equiv 0$. Indeed, we show above that a formula for $\epsilon(t)$ can be obtained for all $f(t)$ provided that eq 5 is used instead of the free evolution for $\tilde{O}$.

In comparison with the classical tracking, this algorithm is a singularity-free noniterative method. In fact, it is a stabilization technique, and unfortunately no control on the convergence time is generally available. However, in the cases considered here and in many other additional situations, we have observed that the time needed for the convergence of the algorithm is of the same order as other methods, e.g., conventional tracking or optimal control. If however, for a given setting, the method has not finished converging at time $T$, two approaches can be proposed. If there are no restrictions on the total time $T$, setting it to a larger value ensures that smaller neighborhoods of the target state will be reached because we know that the procedure only stops when hitting the target. Otherwise, if we deal with a control problem with a fixed time $T$, we can use this algorithm iteratively to get a better result at each step. Using a general formulation of the coherent control theory with a performance index $y(t)$ defined in eq 2, we obtain the following algorithm (a similar algorithm can be written in the density matrix formulation):

1. Use an arbitrary driving field $\epsilon^{(0)}(t)$ for the reference trajectory of different observables $\tilde{O}_{j}^{(0)}$ and the Lyapunov-based method, to obtain a first candidate for the control field $\epsilon^{(1)}(t)$ and a trajectory $\Psi^{(1)}$ of the system for $t \in[0, T]$

$$
\begin{gathered}
\frac{\mathrm{d}}{\mathrm{dt}} \tilde{O}_{j}^{(0)}=i\left[\tilde{O}_{j}^{(0)}, H_{0}-\epsilon^{(0)}(t) \mu\right] \\
\left.\tilde{O}_{j}^{(0)}\right|_{t=T}=O_{j} \quad \text { for } j \in\{1,2, \ldots, N\} \\
i \frac{\partial}{\partial t} \Psi^{(1)}=H_{0} \Psi^{(1)}-\epsilon^{(1)}(t) \mu \Psi^{(1)} \\
\left.\Psi^{(1)}\right|_{t=0}=\Psi_{0}
\end{gathered}
$$

$$
\begin{array}{rl}
\epsilon^{(1)}(t) & =\epsilon^{(0)}(t)+ \\
K & K\left(t,-\sum_{j=1}^{N} \frac{\partial y(t)}{\partial\left\langle\tilde{O}_{j}^{(0)}(t)\right\rangle}\left\langle\Psi^{(1)}(t)\left|\left[\tilde{O}_{j}^{(0)}(t), \mu / i\right]\right| \Psi^{(1)}(t)\right\rangle\right)
\end{array}
$$

2. At the $i$ 's step $(i \geq 1)$, use $\epsilon^{(i)}(t)$ as the reference driving field and find new reference observables $\tilde{O}_{j}^{(i)}$ reaching $O_{j}$ at 
$t=T$. Then, update the control field using the Lyapunov-based method and find the new control field $\epsilon^{(i+1)}(t)$ and the corresponding trajectory $\Psi^{(i+1)}$

$$
\begin{gathered}
\frac{\mathrm{d}}{\mathrm{d} t} \tilde{O}_{j}^{(i)}=i\left[\tilde{O}_{j}^{(i)}, H_{0}-\epsilon^{(i)}(t) \mu\right] \\
\left.\tilde{O}_{j}^{(i)}\right|_{t=T}=O_{j} \quad \text { for } j \in\{1,2, . ., N\} \\
i \frac{\partial}{\partial t} \Psi^{(i+1)}=H_{0} \Psi^{(i+1)}-\epsilon^{(i+1)}(t) \mu \Psi^{(i+1)} \\
\left.\Psi^{(i+1)}\right|_{t=0}=\Psi_{0} \\
K\left(t,-\sum_{j=1}^{N} \frac{\partial y(t)}{\partial\left\langle\tilde{O}_{j}^{(i)}(t)\right\rangle}\left\langle\Psi^{(i+1)}(t)\left|\left[\tilde{O}_{j}^{(i)}(t), \mu / i\right]\right| \Psi^{(i+1)}(t)\right\rangle\right)
\end{gathered}
$$

We can prove that this algorithm has a very convenient monotonic convergence to the target, i.e.

$$
\begin{aligned}
& y\left(\left\{\left\langle\Psi^{(i+1)}(T)\left|O_{j}\right| \Psi^{(i+1)}(T)\right\rangle\right\}_{j=1}^{N}\right) \geq \\
& y\left(\left\{\left\langle\Psi^{(i)}(T)\left|O_{j}\right| \Psi^{(i)}(T)\right\rangle\right\}_{j=1}^{N}\right) \quad \forall i \geq 0
\end{aligned}
$$

Indeed, because $\epsilon^{(i+1)}(t)$ is chosen to have an increasing function $y\left(\left\{\left\langle\Psi^{(i+1)}(t)\left|\tilde{O}_{j}^{(i)}(t)\right| \Psi^{(i+1)}(t)\right\rangle\right\}_{j=1}^{N}\right)$, one must have

$$
\begin{aligned}
& y\left(\left\{\left\langle\Psi^{(i+1)}(T)\left|\tilde{O}_{j}^{(i)}(T)\right| \Psi^{(i+1)}(T)\right\rangle\right\}_{j=1}^{N}\right) \geq \\
& y\left(\left\{\left\langle\Psi^{(i+1)}(0)\left|\tilde{O}_{j}^{i}(0)\right| \Psi^{(i+1)}(0)\right\rangle\right\}_{j=1}^{N}\right) \\
& =y\left(\left\{\left\langle\Psi_{0}\left|\tilde{O}_{j}^{(i)}(0)\right| \Psi_{0}\right\rangle\right\}_{j=1}^{N}\right)= \\
& y\left(\left\{\left\langle\Psi^{(i)}(T)\left|\tilde{O}_{j}^{(i)}(T)\right| \Psi^{(i)}(T)\right\rangle\right\}_{j=1}^{N}\right)
\end{aligned}
$$

where, in the last equality, we have used the fact that the wave functions $\Psi^{(i)}(t)$ and the observables $\tilde{O}_{j}^{(i)}(t)$ evolve with the same coupling field $\epsilon^{(i)}(t)$ and, therefore, the values for $\left\langle\Psi^{(i)}(t)\left|\tilde{O}_{j}^{(i)}(t)\right| \Psi^{(i)}(t)\right\rangle$ remain constant. Finally, $\tilde{O}_{j}^{(i)}(T)=O_{j}$, which yields eq 22 .

\section{Discussion and Conclusion}

This paper presents an extension of the local time control procedure in ref 30 to obtain external fields that maximize a predefined performance index of interest. We have analyzed the previous implementations of the Lyapunov techniques and explained that these methods fail when "trapped" in a state that does not ensure proper optimality of the performance index. We show that considering a general reference field helps in avoiding such phenomena.

Such local control approaches have no control over the final time required to reach the target. When the initial choice of final time $T$ does not ensure reaching the target, two solutions can be suggested: either to simply increase the simulation time (it can only stop at the target!) or, if the time is to be kept fixed, to use iterative procedures. One such procedure that displays convenient monotonic convergence is presented in section 4.

\section{References and Notes}

(1) Assion, A.; Baumert, T.; Bergt, M.; Brinxner, T.; Kiefer, B.; Seyfried, V.; Strehle, M.; Gerber, G. Science 1998, 282, 919.

(2) Bardeen, C.; Yakovlev, V. V.; Wilson, K. R.; Carpenter, S. D.; Weber, P. M.; Warren, W. S. Chem. Phys. Lett. 1997, 280, 151.

(3) Bardeen, C. J.; Yakovlev, V. V.; Squier, J. A.; Wilson, K. R. J. Am. Chem. Soc. 1997, 120, 13023.

(4) Bergt, M.; Brinxner, T.; Kiefer, B.; Strehle, M.; Gerber, G. J. Phys. Chem. A 1999, 103, 10381

(5) Brummer, P. W.; Shapiro, M. Principles of the Quantum Control of Molecular Processes; Wiley-Interscience: New York, 2003.

(6) Chen, Y.; Gross, P.; Ramakrishna, V.; Rabitz, H.; Mease, K. J. Chem. Phys. 1995, 102, 8001-8010.

(7) Geremia, J. M.; Rabitz, H. Phys. Rev. Lett. 2002, 89, 263902-1-4

(8) Gross, P.; Singh, H.; Rabitz, H.; Mease, K.; Huang, G. M. Phys.

Rev. A 1993, 47, 4593 .

(9) Gross, P.; Neuhauser, D.; Rabitz, H. J. Chem. Phys. 1991, 94 , $1158-1166$

(10) Hornung, T.; Motzkus, M.; de Vivie-Riedle, R. J. Chem. Phys. 2001, 115,3105

(11) Judson, R. S.; Rabitz, H. Phys. Rev. Lett. 1992, 68, 1500.

(12) Koslo, R.; Rice, S. A.; Gaspard, P.; Tersigni, S.; Tannor, D. J. Chem Phys. 1989, 139, 201-220.

(13) Levis, R. J.; Menkir, G.; Rabitz, H. Science 2001, 292, 709.

(14) Lewis, H. R.; Riesenfeld, W. B. J. Math. Phys. 1969, 10, 1458

(15) Li, B.; Turinici, G.; Ramakrishna, V.; Rabitz, H. J. Phys. Chem

B. 2002, 106, 8125-8131.

(16) Lu, Z.-M.; Rabitz, H. J. Phys. Chem. 1995, 99, 13731-13735.

(17) Maday, Y.; Turinici, G. J. Chem. Phys. 2003, 118

(18) Mirrahimi, M.; Rouchon, P. In NOLCOS (Symposium on Nonlinear Control Systems), Stuttgart, Germany, 2004.

(19) Mirrahimi, M.; Rouchon, P. In MTNS (Mathematical Theory of Networks and Systems), Leuven, Belgium, 2004.

(20) Ohtsuki, Y.; Kono, H.; Fujimura, Y. J. Chem. Phys. 1998, 109, 9318-9331.

(21) Phan, M. Q.; Rabitz, H. Chem. Phys. 1997, 217, 389-400.

(22) Phan, M. Q.; Rabitz, H. J. Chem. Phys. 1999, 110, 34-41.

(23) Rabitz, H. Science 2003, 299, 525-527.

(24) Rabitz, H.; de Vivie-Riedle, R.; Motzkus, M.; Kompa, K. Science 2000, 288, 824-828.

(25) Rabitz, H.; Smit, M.; Zhu, W. J. Chem Phys. 1999, 110, 1905.

(26) Rabitz, H.; Zhu, W. J. Chem. Phys. 2003, 119, 3619.

(27) Ramakrishna, V.; Salapaka, M.; Dahleh, M.; Rabitz, H. Phys. Rev

A 1995, 51, 960-966.

(28) Rice, S.; Zhao, M. Optimal Control of Quatum Dynamics; Wiley: New York, 2000. Many additional references to the subjects of this paper may also be found here.

(29) Schirmer, S. G.; Girardeau, M. D.; Leahy, J. V. Phys. Rev. A 2000 , 61,012101 .

(30) Sugawara, M. J. Chem. Phys. 2003, 118, 6784-6800

(31) Sugawara, M.; Fujimura, Y. J. Chem. Phys. 1993, 100, 56465655.

(32) Tannor, D.; Kazakov, V.; Orlov, V. In Time Dependent Quantum Molecular Dynamics; Broeckhove, J., Lathouwers, L., Eds.; Plenum: New York, 1992; pp 347-360.

(33) Tersigni, S. H.; Gaspard, P.; Rice, A. J. Chem. Phys. 1990, 93, $1670-1680$.

(34) Turinici, G.; Rabitz, H. Chem. Phys. 2001, 267, 1-9.

(35) Weinacht, T.; Ahn, J.; Bucksbaum, P. Nature 1999, 397, 233.

(36) Zhu, W.; Rabitz, H. J. Chem. Phys. 1998, 109, 385.

(37) Yedder, A. Ben Haj; Le Bris, C.; Atabek, O.; Bandrauk, A Chelkowski, S. Phys. Rev. A 2004, 69, 041802.

(38) Feit, M. D.; Fleck, J. A., Jr.; Steiger, A. J. Comput. Phys. 1982, 47,412 . 